\title{
Planar Cell Polarity in Organ Formation
}

\section{Deborah J Henderson ${ }^{1}$, David A Long ${ }^{2}$ and Charlotte H Dean ${ }^{3 \square}$}

${ }^{1}$ Cardiovascular Research Centre, Institute of Genetic Medicine, Newcastle University, Newcastle upon Tyne, UK. deborah.henderson@newcastle.ac.uk 2Developmental Biology and Cancer Programme, UCL Great Ormond Street Institute of Child Health, London, UK. d.long@ucl.ac.uk

${ }^{3}$ Inflammation, Repair and Development Section, National Heart and Lung Institute, Imperial College, London, UK. c.dean@imperialac.uk

c Corresponding author

Short Title: PCP in organ formation

Keywords: Planar Cell Polarity, morphogenesis, organogenesis, disease

\begin{abstract}
The Planar Cell Polarity (PCP) pathway controls a variety of morphological events across many species. During embryonic development, the PCP pathway regulates coordinated behaviour of groups of cells to direct morphogenetic processes such as convergent extension and collective cell migration. In this review we discuss the increasingly prominent role of the PCP pathway in organogenesis, focusing on the lungs, kidneys and heart. We also highlight emerging evidence that PCP gene mutations are associated with adult diseases.
\end{abstract}

\section{Introduction}

Planar cell polarity (PCP) was originally identified in Drosophila through the study of mutant flies where PCP disruption can be readily observed in the disturbed arrangement of structures such as wing hairs, bristles on the abdomen or ommatidia in the eye. Since then research on PCP has broadened and it is now understood that the key group of 'core' proteins (Vangl, Celsr, Frizzled, Dishevelled and Prickle) which control PCP, operate more widely to regulate the coordinated behaviour of groups of cells by mediating cytoskeleton dynamics. This ability to regulate cytoskeleton organisation lies at the heart of the PCP pathway's roles in organ formation.

In this article we highlight key themes emerging from recent studies of the PCP pathway in mammalian organ development with an emphasis on three 
organs, the lungs, kidneys and heart. Finally we discuss the emerging role of this pathway in human disease.

\section{The PCP pathway}

The exact details of how planar polarity is controlled at the biochemical level are still not entirely clear and are discussed in other reviews [1-3]. In organ formation the most prominent view is that the PCP pathway is initiated when a Wnt ligand binds to a Frizzled (Fz) receptor at the cell surface and this triggers recruitment of Dishevelled (Dvl) to the cell membrane [4]. Subsequently Dvl recruits additional cytoplasmic proteins, such as Dishevelled associated activator of morphogenesis (Daam) proteins, which promote the activation of GTPases and enable cytoskeletal reorganisation via Rho Kinase (ROCK)[5]. This step of Dvl recruitment to the membrane appears to be a key event in PCP signalling. Interestingly, Seo et al., have now shown that Vangl2 can act cell autonomously to promote recruitment of Dvl to the plasma membrane and activate PCP during convergent extension [6]. The authors suggest that Vangl2 mediated recruitment of Dvl to the cell membrane ensures that this protein can be efficiently activated when PCP signalling is triggered e.g. by a Wnt ligand binding to Fz. Additionally, data from Drosophila indicate that PCP is regulated by the asymmetric distribution of different core PCP proteins at the cell membranes and that this is a pre-requisite to establishing planar polarity across fields of cells[3]. This asymmetric distribution of PCP proteins is not always as prominent in vertebrate organogenesis, but has been observed in several organs including the cochlea, skin epidermis, heart and more recently in the papilla of the tongue and kidneys [7-11]. The asymmetric distribution of PCP proteins may be triggered by one or more inputs, e.g. a gradient of Wnt ligand, anisotropic strain caused by fluid flow or morphogenesis itself. In some organs JNK has been identified as an important intermediary in planar polarity output. However, small GTPases can both activate and be activated by JNK[12]; it is therefore not clear if this constitutes a separate downstream pathway that can directly trigger transcription of target genes via Jun family members or whether it is part of the same pathway that also leads to cytoskeletal modification via GTPases [13]. 


\section{Collective cell migration and convergent extension}

Some of the earliest studies of the PCP pathway in mammalian organ formation highlighted its role in skin and kidney development but there is now an increasing list of other organs for which the PCP pathway has been shown to be required, this includes the heart, lungs, eyes, testes, teeth and tongue among others $[7,9,14-19]$. A recurrent theme in organogenesis is that the PCP pathway drives changes to cell behaviours across groups of cells via cytoskeletal reorganisation. Two prominent morphogenetic processes controlled by PCP are collective cell migration and convergent extension, both of which have a key role in organ development (Figure 1a).

PCP mediated convergent extension has been particularly well studied in neural tube closure. Initiation of this event requires convergent extension and mutations in core PCP pathway genes have been shown to disrupt the closure process [20]. The PCP pathway is also required for later stages of neural tube closure where the apical surface of neuroepithelial cells constricts, allowing the cells to become more cone shaped, thus facilitating bending of the tissue required to close the neural tube [20]. Loss of PCP function is associated with neural tube closure defects that result in spina bifida or the severe closure defect, craniorachischisis [20]. Galea and colleagues have now discovered another important link between the PCP pathway and neural tube closure by showing that conditional deletion of Vangl2 in the surface ectoderm throughout neurulation leads to altered biomechanics resulting in caudal spina bifida [21].

\section{PCP and cytoskeleton polarisation}

Morphogenesis, mediated by the actomyosin network, is critical to precisely shape organs for optimal function. In non-muscle cells actomyosin structures are not always polarised, for example in stress fibres, actin is bundled but not polarised. However, for certain functions, one of which is collective cell migration, the actomyosin network must be polarised, therefore it is not surprising that the PCP pathway plays a role in this process $[22,23]$. In migrating cells, polarised features of the actin cytoskeleton include generation of an actin meshwork at the leading edge of the cell, where actin filaments are arranged with the barbed (plus) ends immediately adjacent to the plasma 
membrane, rapid actin polymerisation at the plus ends helps to propel the cell forwards. [24] (Figure 1b). Studies of organ development have also linked the PCP pathway with another constituent of the cytoskeleton, microtubules. Investigations have revealed that microtubules provide a key network through which directional information required for PCP function can be relayed [25]. It appears that microtubules are particularly important for vesicular trafficking of PCP proteins, to facilitate their asymmetric distribution. The microtubule network is also polarised in migrating cells; the centrosome is oriented to a position between the nucleus and the leading edge of the cell and the microtubules are organised with their minus ends towards the centrosome and their plus ends towards the plasma membrane (Figure 1b) [24]. In addition, cilia are comprised of microtubules and it has been recognised for some time that there is a close relationship between the PCP pathway and cilia [26-29]. There are two major types of cilia, primary (non-motile) and motile. Cilia motility depends on the arrangement of microtubules within the appendage and PCP signalling is required for the directional beating of multiple motile cilia in a number of organs [30].

Primary cilia extend from the surface of most mammalian cells and are important for a number of processes including regulation of the cell cycle and transduction of some signalling pathways. Although there is evidence of a strong connection between the PCP pathway and primary cilia, for example, PCP related phenotypes are observed in cilia mutants, this connection is still not entirely clear [31]. Interestingly, one recent investigation in kidneys indicates that PCP contributes to organ formation independently from the primary cilia [11].

\section{PCP and branching morphogenesis}

Two organs where there has been considerable focus on the role of the PCP pathway are the lungs and kidneys. Both of these organs develop by branching morphogenesis, a process that requires extensive cell migration and morphogenesis to generate and precisely shape an arborized network of tubes with a large surface area. Perturbation of the PCP pathway in the lungs and kidneys impairs tissue morphogenesis and results in fewer and misshapen tubes. In fact analysis of mice with mutations in core PCP genes 
shows similar tubule defects in a number of structures generated by branching morphogenesis suggesting that PCP is uniformly required for this process (Figure 2). In fact recent studies indicate that PCP can contribute to the formation of tubular organs in several ways (see below).

\section{Lung}

Lung development initiates by outpouching from the foregut endoderm at embryonic day (E) 9.5 in mouse and week 4 of human gestation. The initial bud grows out into the surrounding mesoderm and divides to form the primordial lung buds. The network of airways is then generated from these initial buds by branching morphogenesis. Subsequent to this, the gasexchanging region of the lungs is generated from the distal ends of the small airways. Cells at the distal tips of the airways begin to differentiate into specialised alveolar epithelial cells (ATI and ATII) and at the same time the tissue thins and airspaces widen to form air sacs. Finally the alveoli form by thinning of the air sac walls and repeated sub divisions (septation) to generate a large surface area optimised for gas exchange.

Mouse mutants of the PCP genes Vangl2, Celsr1 and Scribble all have fewer and misshapen airways[32,33]. Both phalloidin and non-muscle IIA staining revealed significant changes to the distribution of the actin-myosin cytoskeleton from an early stage of lung development (E11.5), indicating that perturbation of the PCP pathway impairs tissue morphogenesis and this disrupts the number and shape of epithelial tubes (airways) that form (Figure 3) $[32,33]$.

Many airway epithelial cells are multiciliated to facilitate sweeping mucus and particles out of the lungs and not down into them. For this function to be performed efficiently, the cilia must be uniformly orientated to enable coordinated beating and unidirectional fluid flow and it had been postulated that PCP was likely to play a role in orientating these motile cilia[14,34]. Studies have now shown that PCP does act to orient motile cilia in the lungs [35] and interestingly, clear asymmetric localisation of core PCP proteins can be observed in tracheal epithelial cells [35]. In addition to misaligned cilia, Vangl1 and Prickle2 mutant tracheal epithelial cells show defective barrier function and diminished regenerative capacity [36]. 
The reduced airway generation caused by PCP dysfunction also impacts on later stages of lung development since the primitive air sacs (saccules) arise from the distal tips of the airways. At embryonic day 18.5, homozygous Celsr1, Vangl2 and Scribble mutant lungs are all hypoplastic and histology shows dense tissue with fewer and smaller airspaces compared to wildtype littermates[32,33].

The PCP pathway has also been shown to be important for alveolar formation, which in the mouse, occurs after birth. Using the Looptail (Vangl2 ${ }^{L p}$ ) mouse as a model of PCP pathway dysfunction, Poobalasingam and colleagues showed marked airspace enlargement in post-natal heterozygous Vangl2Lp lungs both during and after alveologenesis [37]. This was accompanied by disordered actin cytoskeleton distribution and abnormal regulation of the actin-modifying protein cofilin. The altered distribution and regulation of the actin cytoskeleton in the postnatal lungs indicates that as in airway generation, the PCP pathway is required for tissue morphogenesis (septation) during the alveolar stage of lung development.

\section{Kidney}

The formation of the mammalian kidney is initiated around E10.5 in mice when the ureteric bud invades the surrounding metanephric mesenchyme. Similar to the lung, branching morphogenesis is a key process in kidney development facilitating the formation of a highly complex tree of collecting ducts from the ureteric bud. The metanephric mesenchyme differentiates into epithelial structures which form the glomeruli, proximal tubule and loop of Henle components of the nephron [38].

PCP is critical for ureteric bud branching and the growth of the developing kidney. In Vangl2Lp/Lp mutant embryos, the initiation of the ureteric bud is normal but subsequent ureteric bud branching within the metanephros is impaired [39]. Similarly, in studies using optical projection tomography (OPT) and detailed quantitative image analysis (Figure 4), it was found that the ureteric tree of Celsr1Crsh/Crsh mutants was simplified with E13.5 metanephroi containing reduced ureteric bud numbers and branch points than wild-type littermates [40]. Additionally, heterozygous compound mutants in Celsr1 and 
Vangl2 had more rudimentary ureteric trees than those with single heterozygous mutants in either gene indicating an interaction between the two genes. The effect on ureteric branching was more pronounced in the caudal region of the metanephroi in compound heterozygous mice, a finding which may reflect the shortened body axis seen in PCP mutants. Alternatively, there may be differences between rostral and caudal branching in the kidney with Celsr1 transcripts being depleted in the caudal versus rostral portion of E13.5 wildtype kidneys [41]. Analogous to the lung, cytoskeletal rearrangements are critical for ureteric bud branching and $V a n g l 2^{L p / L p}$ kidneys have irregular actin expression suggesting a potential mechanism by which PCP alters branching morphogenesis in the kidney $[40,42]$.

Evidence also implicates PCP in the maturation of the kidney glomerular filtration barrier. PCP genes are expressed in podocytes, the specialised epithelial cells of the glomerulus. Vang/2Lp/Lp [40] and podocyte-specific Vangl2 knock-down kidneys contain smaller and immature glomeruli [43]. In culture, siRNA depletion of Vangl2 reduces the number of podocyte cell projections, decreased actin stress fibres and impaired cell motility [44], whilst PCP activation using Wnt5a increases stress fibre number and enhances nephrin endocytosis [42], a key component of the podocyte slit diaphragm. In contrast,

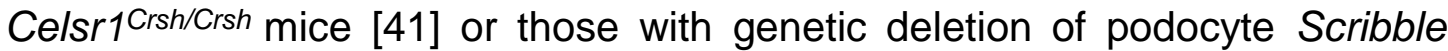
[45] have no changes in glomerular morphology or function, suggesting that not all PCP genes have critical roles in glomeruli.

The morphogenesis of the renal tubules is regulated by a combination of convergence extension and orientated cell division. Vangl2 ${ }^{L p / L p}, \mathrm{Ce} / \mathrm{sr}{ }^{\mathrm{Crsh}} / \mathrm{Crsh}$, double heterozygous Celsr1 $\mathrm{Crsh}_{++} ; \mathrm{Vang} \mathrm{I}^{\mathrm{Lp} /+}$ and $\mathrm{Fz}^{-/-} ; \mathrm{FZ}^{-/-}$double knock-outs all contain dilated tubules $[11,40,41]$. In the tubules of Vangl2 $L / L p$ mice, the asymmetrical distribution of Vangl1, Fz3, Fz6 is impaired suggesting that the specific localisation of PCP components is important for tubular morphogenesis [11]. Furthermore, the tubules of PCP mutants have both defects in convergence extension and orientated cell divisions [11,41]. As defects in orientated cell division are associated with polycystic kidney disease [46] it has been proposed that PCP mutations might lead to 
cystogenesis [47]. However, elegant studies from Kunimoto and colleagues showed that specific tubular deletion of both Vangl1 and Vangl2 did not lead to cyst formation, arguing against a role for PCP in initiating polycystic kidney disease [11].

\section{Heart}

Heart development also involves extensive morphogenesis, but there are considerable differences between the cellular mechanisms of heart development compared with those of the lungs and kidneys. A number of distinct cell populations contribute to the developing cardiovascular system. The primitive heart tube is composed of an endothelial cell tube surrounded by a single layer of cardiomyocytes which is then augmented by the addition of a population of spatially and temporally discrete "second heart field" (SHF) cells that add on to either end to form the right ventricle and the outflow tract [48]. Although it has been known for several years that PCP proteins play an essential role in outflow tract development, it is only recently that the requirement for PCP signalling has begun to be clarified. Using a floxed allele of Vangl2, it was shown that not only are the SHF cells that lengthen the developing outflow tract highly polarised, but that when Vangl2 is deleted specifically within them, using Is/1-Cre, then polarity is disrupted. As a consequence, the cells lose their epithelial characteristics and become disororganised and differentiate prematurely, which ultimately results in a outflow tract shortening and congenital heart defects [49]. Interestingly, very similar defects to those observed when Vangl2 is disrupted i.e. outflow tract lengthening and myocardialisation of the outflow cushions [49-51], are also seen in Prickle1 mutants [52]. This supports roles for PCP signalling in both addition of SHF cells to the forming outflow tract and remodelling of the proximal outflow tract later in development. Related studies have confirmed the importance of PCP signalling to outflow tract development, showing that Wnt5a signalling is essential for the correct incorporation of cells into the epithelium of the SHF and into the outflow tract itself; both loss-of-function and gain-of-function mutations in Wnt5a disrupt outflow tract formation, suggesting that the levels of Wnt ligand are carefully balanced [53,54]. More 
detailed analyses suggested that this may be by controlling the levels of cadherin molecules. PCP effector molecules such as Rac1 have also been confirmed to be important in outflow tract development [55], complementing previous studies that showed that expression of a dominant-negative form of the PCP effector, ROCK, within the Isl1-expressing cells of the SHF, results in malformations of both the venous and arterial poles of the heart [56]. This supports the idea that polarisation of the cells within the SHF is a critical step in their movement into the poles of the heart. Notably, inhibition of canonical Wnt signalling has been shown to be important for activation of the PCP pathway, via the effector Jnk, which modulated SHF proliferation and differentiation [57].

\section{PCP in human disease}

It has been known for some time that mutations in PCP genes can lead to congenital abnormalities such as neural tube defects and heart malformations $[20,52,58]$. Additionally, it has been shown that patients with CELSR1 mutations and spina bifida can have significant renal malformations [41]. However, the last few years have seen an increasing number of reports associating PCP mutations with adult disease. There are now a number of laboratories looking at the PCP pathway in cancer initiation, progression and treatment $[59,60]$. Aside from cancer the PCP pathway has been linked to chronic obstructive pulmonary disease (COPD) [37], glomerular disease [43] in the kidney and susceptibility to fractures [61]. In the next few years, it will be interesting to see if manipulating the PCP pathway could provide opportunities for the treatment of these diseases.

\section{Acknowledgements}

The authors gratefully acknowledge support from the British Heart Foundation to Deborah Henderson (RG/12/15/29935 and $P G / 15 / 46 / 31589)$, The Leverhulme Trust to Charlotte Dean (RPG-2015-226) and The Medical Research Council (MR/J003638/1, MR/P018629/1), Diabetes UK (13/0004763, 15/0005283), Kidney Research UK (RP36/2015), and The National Institute for Health Research Biomedical Research Centre at Great Ormond Street Hospital for Children NHS Foundation Trust and University 
College London to David Long. We thank Thanushiyan Poobalasingam (King's College London) and Laura Yates (Imperial College) for help with illustrations and Eugenia Papakrivopoulou (UCL Institute of Child Health) and Adrian Woolf (University of Manchester) for helpful discussions regarding this work.

\section{Conflict of Interest}

The authors declare no conflict of interest.

\section{References}

1. Humphries AC, Mlodzik M: From instruction to output: Wnt/PCP signaling in development and cancer. Curr Opin Cell Biol 2017, 51:110-116.

2. Campanale JP, Sun TY, Montell DJ: Development and dynamics of cell polarity at a glance. J Cell Sci 2017, 130:1201-1207.

3. Butler MT, Wallingford JB: Planar cell polarity in development and disease. Nat Rev Mol Cell Biol 2017, 18:375-388.

4. Wang Y, Chang H, Rattner A, Nathans J: Frizzled Receptors in Development and Disease. Curr Top Dev Biol 2016, 117:113-139.

5. Papakrivopoulou E, Dean CH, Copp AJ, Long DA: Planar cell polarity and the kidney. Nephrol Dial Transplant 2014, 29:1320-1326.

6. Seo HS, Habas R, Chang C, Wang J: Bimodal regulation of Dishevelled function by Vangl2 during morphogenesis. Hum Mol Genet 2017, 26:2053-2061.

7. Li D, Wang J: Planar Cell Polarity Signaling in Mammalian Cardiac Morphogenesis. Pediatr Cardiol 2018.

8. Geng R, Noda T, Mulvaney JF, Lin VY, Edge AS, Dabdoub A: Comprehensive Expression of Wnt Signaling Pathway Genes during Development and Maturation of the Mouse Cochlea. PLoS One 2016, 11:e0148339.

9. Wang Y, Williams J, Rattner A, Wu S, Bassuk AG, Goffinet AM, Nathans J: Patterning of papillae on the mouse tongue: A system for the quantitative assessment of planar cell polarity signaling. Dev Biol 2016, 419:298-310.

10. Aw WY, Heck BW, Joyce B, Devenport D: Transient Tissue-Scale Deformation Coordinates Alignment of Planar Cell Polarity Junctions in the Mammalian Skin. Curr Biol 2016, 26:2090-2100.

11. Kunimoto K, Bayly RD, Vladar EK, Vonderfecht T, Gallagher AR, Axelrod JD: Disruption of Core Planar Cell Polarity Signaling Regulates Renal Tubule Morphogenesis but Is Not Cystogenic. Curr Biol 2017, 27:31203131 e3124.

12. Davis RJ: Signal transduction by the JNK group of MAP kinases. Cell 2000, 103:239-252.

13. Schlessinger K, Hall A, Tolwinski N: Wnt signaling pathways meet Rho GTPases. Genes Dev 2009, 23:265-277. 
14. Yates LL, Dean CH: Planar polarity: A new player in both lung development and disease. Organogenesis 2011, 7:209-216.

15. Wu Z, Epasinghe DJ, He J, Li L, Green DW, Lee MJ, Jung HS: Altered tooth morphogenesis after silencing the planar cell polarity core component, Vangl2. Cell Tissue Res 2016, 366:617-621.

16. Findlay AS, Panzica DA, Walczysko P, Holt AB, Henderson DJ, West JD, Rajnicek AM, Collinson JM: The core planar cell polarity gene, Vangl2, directs adult corneal epithelial cell alignment and migration. $R$ Soc Open Sci 2016, 3:160658.

17. Chen H, Mruk DD, Lee WM, Cheng CY: Planar Cell Polarity (PCP) Protein Vangl2 Regulates Ectoplasmic Specialization Dynamics via Its Effects on Actin Microfilaments in the Testes of Male Rats. Endocrinology 2016, 157:2140-2159.

18. Chen J, Chuong CM: Patterning skin by planar cell polarity: the multitalented hair designer. Exp Dermatol 2012, 21:81-85.

19. Schnell U, Carroll TJ: Planar cell polarity of the kidney. Exp Cell Res 2016, 343:258-266.

20. Nikolopoulou E, Galea GL, Rolo A, Greene ND, Copp AJ: Neural tube closure: cellular, molecular and biomechanical mechanisms. Development 2017, 144:552-566.

21. Galea GL, Nychyk O, Mole MA, Moulding D, Savery D, Nikolopoulou E, Henderson DJ, Greene NDE, Copp AJ: Vangl2 disruption alters the biomechanics of late spinal neurulation leading to spina bifida in mouse embryos. Dis Model Mech 2018, 11.

22. Mayor R, Etienne-Manneville $S$ : The front and rear of collective cell migration. Nat Rev Mol Cell Biol 2016, 17:97-109.

23. Munoz-Soriano V, Belacortu Y, Paricio N: Planar cell polarity signaling in collective cell movements during morphogenesis and disease. Curr Genomics 2012, 13:609-622.

24. Rodriguez OC, Schaefer AW, Mandato CA, Forscher P, Bement WM, Waterman-Storer CM: Conserved microtubule-actin interactions in cell movement and morphogenesis. Nat Cell Biol 2003, 5:599-609.

25. Matis M, Russler-Germain DA, Hu Q, Tomlin CJ, Axelrod JD: Microtubules provide directional information for core PCP function. Elife 2014, 3:e02893.

26. Chu CW, Ossipova O, Ioannou A, Sokol SY: Prickle3 synergizes with Wtip to regulate basal body organization and cilia growth. Sci Rep 2016, 6:24104.

27. Carvajal-Gonzalez JM, Roman AC, Mlodzik M: Positioning of centrioles is a conserved readout of Frizzled planar cell polarity signalling. Nat Commun 2016, 7:11135.

28. Ohata S, Nakatani J, Herranz-Perez V, Cheng J, Belinson H, Inubushi T, Snider WD, Garcia-Verdugo JM, Wynshaw-Boris A, Alvarez-Buylla A: Loss of Dishevelleds disrupts planar polarity in ependymal motile cilia and results in hydrocephalus. Neuron 2014, 83:558-571.

29. Boutin C, Labedan P, Dimidschstein J, Richard F, Cremer H, Andre P, Yang Y, Montcouquiol M, Goffinet AM, Tissir F: A dual role for planar cell polarity genes in ciliated cells. Proceedings of the National Academy of Sciences of the United States of America 2014, 111:E3129-3138. 
30. Wallingford JB: Planar cell polarity signaling, cilia and polarized ciliary beating. Curr Opin Cell Biol 2010, 22:597-604.

31. Berbari NF, O'Connor AK, Haycraft CJ, Yoder BK: The primary cilium as a complex signaling center. Curr Biol 2009, 19:R526-535.

32. Yates LL, Schnatwinkel C, Murdoch JN, Bogani D, Formstone CJ, Townsend S, Greenfield A, Niswander LA, Dean CH: The PCP genes Celsr1 and Vangl2 are required for normal lung branching morphogenesis. Human molecular genetics 2010, 19:2251-2267.

33. Yates LL, Schnatwinkel C, Hazelwood L, Chessum L, Paudyal A, Hilton H, Romero MR, Wilde J, Bogani D, Sanderson J, et al.: Scribble is required for normal epithelial cell-cell contacts and lumen morphogenesis in the mammalian lung. Dev Biol 2013, 373:267-280.

34. Lewis J, Davies A: Planar cell polarity in the inner ear: how do hair cells acquire their oriented structure? J Neurobiol 2002, 53:190-201.

35. Vladar EK, Bayly RD, Sangoram AM, Scott MP, Axelrod JD: Microtubules enable the planar cell polarity of airway cilia. Curr Biol 2012, 22:22032212.

36. Vladar EK, Nayak JV, Milla CE, Axelrod JD: Airway epithelial homeostasis and planar cell polarity signaling depend on multiciliated cell differentiation. JCI Insight 2016, 1.

37. Poobalasingam T, Yates LL, Walker SA, Pereira M, Gross NY, Ali A, KolatsiJoannou M, Jarvelin MR, Pekkanen J, Papakrivopoulou E, et al.:

Heterozygous Vangl2(Looptail) mice reveal novel roles for the planar cell polarity pathway in adult lung homeostasis and repair. Dis Model Mech 2017, 10:409-423.

38. Costantini F, Kopan R: Patterning a complex organ: branching morphogenesis and nephron segmentation in kidney development. Dev Cell 2010, 18:698-712.

39. Yates LL, Papakrivopoulou J, Long DA, Goggolidou P, Connolly JO, Woolf AS, Dean $\mathrm{CH}$ : The planar cell polarity gene Vangl2 is required for mammalian kidney-branching morphogenesis and glomerular maturation. Hum Mol Genet 2010, 19:4663-4676.

40. Yates LL, Papakrivopoulou J, Long DA, Goggolidou P, Connolly JO, Woolf AS, Dean $\mathrm{CH}$ : The planar cell polarity gene Vangl 2 is required for mammalian kidney-branching morphogenesis and glomerular maturation. Human molecular genetics 2010.

41. Brzoska HL, d'Esposito AM, Kolatsi-Joannou M, Patel V, Igarashi P, Lei Y, Finnell RH, Lythgoe MF, Woolf AS, Papakrivopoulou E, et al.: Planar cell polarity genes Celsr 1 and Vangl2 are necessary for kidney growth, differentiation, and rostrocaudal patterning. Kidney Int 2016, 90:1274-1284.

42. Babayeva S, Rocque B, Aoudjit L, Zilber Y, Li J, Baldwin C, Kawachi H, Takano $\mathrm{T}$, Torban E: Planar cell polarity pathway regulates nephrin endocytosis in developing podocytes. J Biol Chem 2013, 288:2403524048.

43. Rocque BL, Babayeva S, Li J, Leung V, Nezvitsky L, Cybulsky AV, Gros P, Torban E: Deficiency of the planar cell polarity protein Vangl2 in podocytes affects glomerular morphogenesis and increases susceptibility to injury. J Am Soc Nephrol 2015, 26:576-586. 
44. Babayeva S, Zilber Y, Torban E: Planar cell polarity pathway regulates actin rearrangement, cell shape, motility, and nephrin distribution in podocytes. Am J Physiol Renal Physiol 2011, 300:F549-560.

45. Hartleben B, Widmeier E, Wanner N, Schmidts M, Kim ST, Schneider L, Mayer $\mathrm{B}$, Kerjaschki D, Miner JH, Walz G, et al.: Role of the polarity protein Scribble for podocyte differentiation and maintenance. PLoS One 2012, 7:e36705.

46. Fischer E, Legue E, Doyen A, Nato F, Nicolas JF, Torres V, Yaniv M, Pontoglio M: Defective planar cell polarity in polycystic kidney disease. Nature genetics 2006, 38:21-23.

47. Luyten A, Su X, Gondela S, Chen Y, Rompani S, Takakura A, Zhou J: Aberrant regulation of planar cell polarity in polycystic kidney disease. $\mathrm{J} \mathrm{Am}$ Soc Nephrol 2010, 21:1521-1532.

48. Kelly RG, Buckingham ME, Moorman AF: Heart fields and cardiac morphogenesis. Cold Spring Harb Perspect Med 2014, 4.

49. Ramsbottom SA, Sharma V, Rhee HJ, Eley L, Phillips HM, Rigby HF, Dean C, Chaudhry B, Henderson DJ: Vangl2-Regulated Polarisation of Second Heart Field-Derived Cells Is Required for Outflow Tract Lengthening during Cardiac Development. PLoS genetics 2014, 10:e1004871.

50. Phillips HM, Murdoch JN, Chaudhry B, Copp AJ, Henderson DJ: Vangl2 acts via RhoA signaling to regulate polarized cell movements during development of the proximal outflow tract. Circ Res 2005, 96:292-299.

51. Henderson DJ, Conway SJ, Greene ND, Gerrelli D, Murdoch JN, Anderson RH, Copp AJ: Cardiovascular defects associated with abnormalities in midline development in the Loop-tail mouse mutant. Circulation research 2001, 89:6-12.

52. Gibbs BC, Damerla RR, Vladar EK, Chatterjee B, Wan Y, Liu X, Cui C, Gabriel GC, Zahid M, Yagi H, et al.: Prickle1 mutation causes planar cell polarity and directional cell migration defects associated with cardiac outflow tract anomalies and other structural birth defects. Biol Open 2016, 5:323-335.

53. Li D, Sinha T, Ajima R, Seo HS, Yamaguchi TP, Wang J: Spatial regulation of cell cohesion by Wnt5a during second heart field progenitor deployment. Dev Biol 2016, 412:18-31.

54. Sinha T, Lin L, Li D, Davis J, Evans S, Wynshaw-Boris A, Wang J: Mapping the dynamic expression of Wnt11 and the lineage contribution of Wnt11-expressing cells during early mouse development. Dev Biol 2015, 398:177-192.

55. Leung C, Liu Y, Lu X, Kim M, Drysdale TA, Feng Q: Rac1 Signaling Is Required for Anterior Second Heart Field Cellular Organization and Cardiac Outflow Tract Development. J Am Heart Assoc 2015, 5.

56. Phillips HM, Mahendran P, Singh E, Anderson RH, Chaudhry B, Henderson DJ: Neural crest cells are required for correct positioning of the developing outflow cushions and pattern the arterial valve leaflets. Cardiovasc Res 2013, 99:452-460.

57. Schmeckpeper J, Verma A, Yin L, Beigi F, Zhang L, Payne A, Zhang Z, Pratt RE, Dzau VJ, Mirotsou M: Inhibition of Wnt6 by Sfrp2 regulates adult cardiac progenitor cell differentiation by differential modulation of Wnt pathways. J Mol Cell Cardiol 2015, 85:215-225. 
58. Qiao X, Liu Y, Li P, Chen Z, Li H, Yang X, Finnell RH, Yang Z, Zhang T, Qiao B, et al.: Genetic analysis of rare coding mutations in CELSR1-3 in Chinese Congenital Heart and Neural Tube Defects. Clin Sci (Lond) 2016.

59. Daulat AM, Borg JP: Wnt/Planar Cell Polarity Signaling: New Opportunities for Cancer Treatment. Trends Cancer 2017, 3:113-125.

60. VanderVorst K, Hatakeyama J, Berg A, Lee H, Carraway KL, 3rd: Cellular and molecular mechanisms underlying planar cell polarity pathway contributions to cancer malignancy. Semin Cell Dev Biol 2017.

61. Orriss IR, Lanham S, Savery D, Greene NDE, Stanier P, Oreffo R, Copp AJ, Galea GL: Spina bifida-predisposing heterozygous mutations in Planar Cell Polarity genes and Zic2 reduce bone mass in young mice. Sci Rep 2018, 8:3325.

\section{Figure Legends}

Figure 1a The PCP pathway regulates collective cell migration and convergent extension.

Collective cell migration enables the coordinated movement of groups of cells in the same direction. Actin filled protrusions, such as lamellipodia, form at the leading edge of cells to drive migration. Convergent extension involves the intercalation of cells, resulting in narrowing along one axis, medio-laterial (M), medial, (L) lateral and subsequent extension of the tissue along the perpendicular axis, anterior-posterior $(A)$, anterior, $(P)$ posterior. Red arrows indicate direction of cell movement; thin black arrows show axes.

Figure 1b Features of a polarised cytoskeleton in a migrating cell.

Migrating cells exhibit an actin meshwork at the leading edge. Microfilaments are rapidly polarised at the barbed (plus) ends, adjacent to the plasma membrane; this helps to propel the cell forward. Microtubules are also polarised with their minus ends (-) towards the centrosome, which lies between the nucleus (blue) and the leading edge, and their plus ends (+) towards the plasma membrane. Black arrow indicates the direction of cell migration.

Figure 2 Mutations in core PCP genes Vangl2 and Celsr1 disrupt branching morphogenesis. 
Lungs $(A, B, C)$ Kidneys $(D, E)$ Lacrimal glands $(F, G)$ and Salivary glands $(H, I)$ are dysmorphic in E18.5 Celsr ${ }^{\text {Crsh/Crsh }}$ and Vangl2Lp/Lp mice.

Figure 3 Schematic representation of PCP induced collective cell migration in lung branching morphogenesis.

PCP proteins are asymmetrically localised (yellow) at one side of the plasma membrane in epithelial cells to facilitate bud outgrowth during branching morphogenesis, cell cortex is shown in red. The planar polarity axis is orthogonal to the apical (green)- basal (blue) axis. Direction of bud growth/cell migration is indicated by black arrows.

Figure 4 Assessment of ureteric bud branching in mice with mutations in PCP genes.

Images of whole metanephroi from E13.5 wild-type (A), Celsr1Crsh/t (B), Celsr1Crsh/Crsh (C), Vangl2L//+ (D) and Celsr1Crsh/+;Vangl2Lp/+ $(\mathrm{E})$ mice stained with calbindin-D28K and visualised by optical projection tomography[41].

\section{References to highlight}

2. ++ Campanale et al.- A comprehensive review on cell polarity mechanisms and how polarity is linked with cell dynamics.

3. + Butler et al.- This review highlights the links between the PCP pathway and disease.

6. ++ Seo et al. Describes bimodal regulation of dishevelled including Vangl2 induced recruitment to the cell membrane.

31. ++ Vladar et al. 2016 -This paper shows the PCP pathway is required for differentiation of multi-ciliated airway epithelial cells. The authors describe a role for planar cell polarity in airway homeostasis and show disrupted barrier function in PCP mutant airway epithelia. In addition they show defective PCP in airway epithelial cells obtained from human patients with lung disease. 
14. + Yates and Dean 2011- A review of what was known about PCP in lung development in 2011, this highlights how far we have come in understanding $\mathrm{PCP}$ in organ development.

21. ++ Galea et al. 2018- This manuscript reports a link between Planar polarized events and biomechanics in neural tube closure. The findings may explain why a subset of VANGL2 mutations, in humans, are associated with defects caused by perturbation of the very late stages of neural tube closure (closure 5).

23. +Munoz-Soriano 2012- A concise review of PCP in collective cell migration.

36. ++Brzoska et al 2016-This paper used sophisticated image analysis to establish a role for Celsr1 in ureteric tree growth. The authors also found an interaction between Celsr1 and Vangl2 in ureteric tree growth, most marked in the caudal compartment of the kidney.

11. ++Kunimoto et al. 2017-These authors found tubular dilation in multiple mice models with defective PCP. This was associated with disruption in the asymmetric distribution of PCP components and alterations in convergence extension and orientated cell division. However, knock-down of PCP proteins did not lead to the formation of cysts.

44. ++Ramsbottom - The first paper to show how disruption of PCP signalling specifically in the second heart field leads to outflow tract defects

49. +Sinha - Shows that Wnt11 plays sequential roles in the first and second heart fields 